\title{
Diverse Psychotropic Substances Detected in Drug and Paraphernalia Samples Submitted to Drug Checking Services in Toronto, Ontario, Canada, October 2019-April 2020
}

\section{Kristy Scarfone}

St Michael's Hospital Li Ka Shing Knowledge Institute

Nazlee Maghsoudi

St Michael's Hospital Li Ka Shing Knowledge Institute

Karen McDonald

St Michael's Hospital Li Ka Shing Knowledge Institute

Cristiana Stefan

CAMH: Centre for Addiction and Mental Health

Daniel R Beriault

St Michael's Hospital

\section{Ernest Wong}

CAMH: Centre for Addiction and Mental Health

\section{Mark Evert}

St Michael's Hospital

\section{Shaun Hopkins}

Toronto Public Health

\section{Peter Leslie}

Toronto Harm Reduction Alliance

\section{Tara Marie Watson}

CAMH: Centre for Addiction and Mental Health

Dan Werb ( $\triangle$ Daniel.Werb@unityhealth.to)

University of California San Diego https://orcid.org/0000-0003-0614-9386

\section{Brief report}

Keywords: drug overdose, fentanyl, etizolam, flualprazolam, synthetic cannabinoid

Posted Date: July 26th, 2021

DOI: https://doi.org/10.21203/rs.3.rs-694379/v1 
License: (c) (i) This work is licensed under a Creative Commons Attribution 4.0 International License. Read Full License 


\section{Abstract}

\section{Background}

The opioid overdose crisis has generated innovative harm reduction and drug market monitoring strategies. In Toronto, Ontario, Canada, a multi-site drug checking service (DCS) pilot project was launched in October 2019. The project provides people who use drugs with information on the chemical composition of their substances, thereby increasing their capacity to make more informed decisions about their drug use and avoid overdose. DCS also provides real-time market monitoring to identify trends in the unregulated drug supply.

\section{Methods}

Sample data were obtained through analyses of drug and used paraphernalia samples submitted anonymously and free of charge to DCS in downtown Toronto from October 10, 2019 to April 9, 2020, representing the first six months of DCS implementation. Analyses were conducted in clinical laboratories using ultra high performance liquid chromatography-high resolution mass spectrometry (UPLC-HRMS), and liquid chromatography or gas chromatography-mass spectrometry (LC-MS, GC-MS) techniques.

\section{Results}

Overall, 555 samples were submitted, with $49 \%$ (271) of samples that were found to contain high-potency opioids, of which $87 \%$ (235) also contained stimulants. Benzodiazepines or related drugs were also found in $21 \%$ (116) of all samples, and synthetic cannabinoids in $1 \%$ (7) of all samples. Negative effects (including overdose) were reported for $12 \%$ (69) of samples submitted for analysis.

\section{Conclusions}

Toronto's DCS identified a range of high-potency opioids with stimulants, benzodiazepines and related drugs, and a synthetic cannabinoid, AMB-FUBINACA. This information can inform a range of evidenceinformed overdose prevention efforts.

\section{Introduction}

The opioid overdose crisis is worsening throughout much of North America, particularly in Canada and the United States. ${ }^{1,2}$ Between January 2016 and March 2020, more than 16,364 Canadians died from apparent opioid-related overdoses. ${ }^{3}$ Recent estimates suggest that 1 in 6 deaths of Ontarians aged 25-34 years is related to opioid overdose. ${ }^{4}$ Moreover, the incidence of fatal overdose has increased in Ontario since the imposition of COVID-19 restrictions in March 2020. ${ }^{5}$

The dynamic uncertainty in the unregulated drug market has generated innovative harm reduction approaches in Canada, including the implementation of drug checking services (DCS) tailored for structurally vulnerable people who use drugs. ${ }^{6}$ Originating in California amid the counterculture 
movement in the 1960 s and early $1970 \mathrm{~s}^{7}$ and having later gained popularity in the 1990s in European nightlife and dance settings ${ }^{8}$, DCS provide chemical analysis of substances to clients while contributing data to drug market monitoring. ${ }^{9}$

A multi-site DCS pilot was launched in Toronto, Ontario, Canada in October 2019. ${ }^{10}$ Intake sites are colocated with supervised consumption services (SCS) at multiple harm reduction agencies. The project prioritizes providing structurally vulnerable (i.e., socially and economically marginalized) people who use drugs with information about the composition of their substances, thereby increasing their capacity to avoid overdose.

This report presents early trends of samples analyzed within the first six months of DCS implementation in Toronto (October 10, 2019-April 9, 2020). We sought to identify the prevalence of high-potency opioids in combination with stimulants, benzodiazepines and related drugs, and synthetic cannabinoids. We also present data on reported negative effects of samples.

\section{Methods}

The protocol and rationale for the evaluation of Toronto's DCS has previously been described. ${ }^{10}$ In brief, we are evaluating the impact of Toronto's DCS on overdose and related risk behaviors among clients and its capacity to identify trends in the chemical composition of Toronto's unregulated drug supply. The results presented herein were obtained through chemical analyses of samples submitted anonymously and free of charge to DCS. Samples are analyzed using ultra high performance liquid chromatographyhigh resolution mass spectrometry (UPLC-HRMS), and liquid and gas chromatography-mass spectrometry (LC-MS, GC-MS) techniques, which are the gold standards in forensic drug analysis. ${ }^{11}$

Results are provided within one to two business days. Aggregated results are publicly shared online (drugchecking.cdpe.org). ${ }^{12}$ The analytical results are supplemented by data from surveys administered during sample collection, including drug expectation, the reuse of paraphernalia, and negative effects post-use.

\section{Results}

Overall, 555 samples were submitted for analysis by DCS, among which $63 \%(n=349)$ were substances (i.e., liquid, pill, powder) and 37\% ( $n=206)$ paraphernalia (i.e., cooker, filter)., $10 \%(n=21)$ of which reportedly reused. The number of samples stratified by type and category of drug detected are presented in Figure 1.

\section{Negative effects}

Survey responses indicate that $12 \%(n=69)$ of all samples submitted for analysis by the DCS were associated with negative effects. Of these samples, survey responses report that: $54 \%(n=37)$ were 
implicated in an overdose, $29 \%(n=20)$ were associated with feeling unwell, $7 \%(n=5)$ were associated with drowsiness, $7 \%(n=5)$ had a stronger effect than anticipated, and $3 \%(n=2)$ had little or no effect.

\section{Expected opioids and detected contents}

An opioid was expected in 49\% ( $n=272)$ of all samples. Fentanyl was expected most often, in $94 \%$ $(n=256)$ of all expected opioids. Figure 2 depicts the detected drugs found in samples expected to contain opioids upon submission, including unexpected high potency opioids, unexpected benzodiazepines-type drugs, and unexpected synthetic cannabinoids.

\section{Presence of stimulants with high-potency opioids}

A high potency opioid (i.e., fentanyl, fentanyl-related drugs, carfentanil) was detected in $49 \%(n=271)$ of all samples submitted for analysis by the DCS. In $87 \%(n=235)$ of these samples where a high potency opioid was detected, one or more stimulants were found in combination. Of this fraction of samples, a high-potency opioid (i.e., fentanyl, carfentanil) was expected in 216 samples, or 92\%. Caffeine was the most commonly co-detected stimulant in $79 \%(n=215)$. The most frequent combinations detected were fentanyl with caffeine $(n=215)$, with cocaine $(n=61)$, with methamphetamine $(n=43)$, and with cocainerelated drugs such as anhydroecgonine methyl ester (AEME), benzoylecgonine, and methylecgonine $(n=34)$. In all samples where a high potency was detected with at least one stimulant, the stimulant was not reported to be an expected substance upon submission and are thereby appearing unexpected in expected high potency opioid samples.

\section{Presence of benzodiazepine-type drugs}

At least one benzodiazepine-type drug was detected in $21 \%(n=116)$ of all samples submitted for analysis. Of these samples, $67 \%(n=78)$ contained only one benzodiazepine and $33 \%(n=38)$ contained two or more. Majority of the detected benzodiazepines were found unexpectedly in submitted samples; only $13 \%(n=15)$ were expected to contain benzodiazepines. Fentanyl was expected in $80 \%(n=93)$, and various other drugs in the remaining $7 \%(n=8)$. The primary benzodiazepine-type drugs detected were etizolam ( $n=95)$, flualprazolam in $(n=46)$, alprazolam (Xanax) $(n=9)$, and flubromazolam $(n=4)$. Samples containing benzodiazepine-type drugs account for $21 \%(n=24)$ of samples associated with a negative effect, 22 of which were implicated in an overdose.

\section{Presence of synthetic cannabinoids}

AMB-FUBINACA was the only synthetic cannabinoid detected. It was found in $1 \%(n=7)$ of all samples, all of which were expected to contain fentanyl.

\section{Discussion}

Across a six-month period in Toronto, a large proportion of samples submitted to DCS contained highpotency opioids presenting with unexpected stimulants. We also detected various benzodiazepine-type 
drugs and a synthetic cannabinoid, AMB-FUBINACA.

The prevalence of high-potency opioids is noteworthy given that they carry increased risk for overdose. Caffeine was the most common stimulant co-presenting with high-potency opioids, which is consistent with other settings. ${ }^{13}$ To our knowledge no published scientific literature exists regarding the impact of intravenous administration of caffeine in a non-clinical setting. Further research is required to clinically assess the potential contribution of caffeine injection to overdose or other adverse events.

The detection of various benzodiazepine-type drugs is significant given the risk of overdose that they present, especially when unexpected and combined with opioids. Benzodiazepines elicit sedative and anxiolytic effects that, when combined with other central nervous system depressants, may amplify cardiovascular and respiratory depression and contribute to overdose severity. ${ }^{14}$ Compounding this issue is the lack of reliable and effective antagonist therapies to reverse benzodiazepine overdose outside of clinical settings. ${ }^{15}$ Additionally, increased use of benzodiazepines may increase tolerance and the potential for withdrawal, especially if individuals are not aware that they are using these substances. This issue therefore demands greater urgency as it appears to be contributing to overdose mortality in Toronto and elsewhere. ${ }^{16}$ For instance, on February 26,2020 , a notable spike in overdoses (16 in seven hours) in Toronto was associated with drug samples containing large proportions of flualprazolam along with fentanyl and caffeine. ${ }^{17}$

We also detected AMB-FUBINACA, an ultra-potent synthetic cannabinoid that can be up to hundreds of times more potent than D9-tetrahydrocannabinol. ${ }^{18}$ Synthetic cannabinoid exposure may cause adverse effects including neuropsychiatric, cardiovascular, and renal impairment ${ }^{18}$, but the effects of coadministration of AMB-FUBINACA and other central nervous system depressants (e.g., benzodiazepines, opioids) remain largely unknown. It is therefore critical to share information on their presentation in unregulated drug markets with people who use drugs, harm reduction workers, and clinicians.

There are limitations to the capacity of Toronto's DCS to provide a comprehensive analytical assessment of the unregulated drug supply. Inherent limitations of mass spectrometry analysis include accessibility, cost, destruction of the sample during analysis, contaminants obscuring data outputs, the speed at which results can be provided to clients, and the inability to detect certain non-drug compounds (e.g., metals, pesticides, inorganic salts, sugars). ${ }^{11}$ With regards to sample composition, the results may not represent the entire supply that samples are taken from, and reused paraphernalia may contain substances from multiple uses.

\section{Conclusions}

This report briefly highlights the presence of highly-potent substances and combinations thereof in Toronto's unregulated drug market. Timely data and public health alerts are critical to inform people who may experience health harms from drug use and demonstrate the value of DCS for drug market 
monitoring. Examples of such alerts were issued by Toronto Public Health regarding noteworthy trends in Toronto's unregulated drug supply. ${ }^{12}$

However, DCS alone are insufficient to prevent overdose and mortality arising from an unregulated drug supply, especially among persons without access to medication-assisted treatment for drug dependence or other standard-dose regimens as a result of drug policies (e.g., drug criminalization) that amplify social marginalization. ${ }^{19}$ Quantifying the persisting contaminated drug supply and addressing it with policy solutions such as safer supply or other standard-dose modalities is therefore urgently needed. ${ }^{20}$

As the global COVID-19 pandemic continues, drug trafficking patterns and supply are likely to remain dynamic. ${ }^{21}$ This increases the need to sustain and expand DCS to prevent overdose, and to monitor drug market fluctuations.

\section{Declarations}

\section{Ethics Approval and Consent to Participate:}

Research Ethics Board approval has been obtained for this research study from Unity Health Toronto, Toronto Public Health, Centre for Addiction and Mental Health, and University of Toronto.

\section{Consent for Publication:}

While informed consent is obtained from participants for this study, the present manuscript does not publish any identifiable personal data or images.

\section{Availability of Data and Material:}

A selection of the data presented herein is available online at: https://drugchecking.cdpe.org/

\section{Competing Interests:}

The authors declare that they have no conflicts of interest.

\section{Funding:}

Toronto's Drug Checking Service is supported by an operating grant from Health Canada's Substance Use and Addictions Program (Award \#1718-HQ-000027, Recipient: Dan Werb, PhD) and the St. Michael's Hospital Foundation. Dan Werb is supported by the Canadian Institutes of Health Research via a New Investigator Award, and the Ontario Ministry of Research, Innovation and Science via an Early Researcher Award. Nazlee Maghsoudi is supported by a CIHR Vanier Canada Graduate Scholarship.

\section{Author's Contributions:}


Laboratory analysisdata was generated by CS, DRB, EW, and ME.DW, NM, and KM conceptualized the paper. KS drafted the manuscript and figures. All authors reviewed and provided substantive input on the manuscript. All authors read and approved the final manuscript.

\section{Acknowledgements:}

We wish to acknowledge our partnering harm reduction agencies - Parkdale Queen West Community Health Centre (Queen West site), South Riverdale Community Health Centre, and The Works at Toronto Public Health, and partnering clinical laboratories (Centre for Addiction and Mental Health and St.

Michael's Hospital). We thank the members of the Community Advisory Board for Toronto's DCS for their guidance. We also thank those who trusted us with their samples and provided feedback on the service.

Toronto's DCS is supported by an operating grant from Health Canada's Substance Use and Addictions Program and the St. Michael's Hospital Foundation. Dan Werb is supported by the Canadian Institutes of Health Research via a New Investigator Award, and the Ontario Ministry of Research, Innovation and Science via an Early Researcher Award. Nazlee Maghsoudi is supported by a CIHR Vanier Canada Graduate Scholarship.

We acknowledge that the land on which we conduct our research is the traditional territory of many nations including the Mississaugas of the Credit, the Anishinaabe, the Chippewa, the Haudenosaunee, and the Wendat peoples, now also home to many diverse First Nations, Inuit, and Métis peoples.

\section{References}

1. U .S . Drug Enforcement Administration, Diversion Control Division. National Forensic Laboratory Information System: NFLIS-Drug 2019 Midyear Report [Internet]. Springfield,VA; 2020 [cited May 2021]. https://www.nflis.deadiversion.usdoj.gov:8443/nflisdata/docs/NFLIS-

DRUG_2019_Annual_Report.pdf\#search=2019\%20Midyear

2. Payer, D.E., Young, M.M., Maloney-Hall, B., Mill, C., Leclerc, P., Buxton, J., \& the Canadian Community Epidemiology Network on Drug Use, the National Drug Checking Working Group. Adulterants, contaminants and co-occurring substances in drugs on the illegal market in Canada: An analysis of data from drug seizures, drug checking and urine toxicology [Internet]. Ottawa, ON: Canadian Centre on Substance Use and Addiction; 2020 [cited May 2021].

https://www.ccsa.ca/sites/default/files/2020-04/CCSA-CCENDU-Adulterants-Contaminants-Cooccurring-Substances-in-Drugs-Canada-Report-2020-en.pdf.

3. Public Health Agency of Canada; Special Advisory Committee on the Epidemic of Opioid Overdoses. Opioids and stimulant-related Harms in Canada [Internet]. Ottawa, ON: Government of Canada Health Infobase; 2020 [cited May 2021]. https://health-infobase.canada.ca/substance-relatedharms/opioids-stimulants.

4. Gomes T, Greaves S, Tadrous M, Mamdani MM, Paterson JM, Juurlink DN. Measuring the burden of opioid-related mortality in Ontario, Canada. Journal of addiction medicine. 2018 Sep;12(5):418. 
5. Gomes T, Kitchen SA, Murray R. Measuring the Burden of Opioid-Related Mortality in Ontario, Canada, During the COVID-19 Pandemic. JAMA Network Open. 2021 May 3;4(5):e2112865-.

6. House of Commons of Canada, Standing Committee on Health. REPORT AND RECOMMENDATIONS ON THE OPIOID CRISIS IN CANADA [Internet]. Ottawa, ON: Government of Canada Publications; 2016 [cited May 2021]. https://publications.gc.ca/collections/collection_2016/parl/xc62-1/XC62-1-1-4216-eng.pdf

7. Renfroe CL. MDMA on the street: Analysis Anonymous ${ }^{\circledR}$. Journal of psychoactive drugs. 1986 Oct 1;18(4):363-9.

8. Smit-Rigter L, Van der Gouwe D. The Drugs Information and Monitoring System (DIMS). Factsheet on drug checking in the Netherlands. Utrecht, NL: Trimbos Institute. 2019.

9. Ontario Agency for Health Protection and Promotion (Public Health Ontario), Leece P. Evidence Brief: Evidence on drug checking services as a harm reduction intervention [Internet]. Toronto, ON: Queen's Printer for Ontario; 2017 [cited May 2021].

https://www.publichealthontario.ca/-/media/documents/E/2017/eb-drug-checking.pdf.

10. Maghsoudi N, McDonald K, Stefan C, Beriault DR, Mason K, Barnaby L, Altenberg J, MacDonald RD, Caldwell J, Nisenbaum R, Leece P. Evaluating networked drug checking services in Toronto, Ontario: study protocol and rationale. Harm reduction journal. 2020 Dec;17(1):1-0.

11. Harper L, Powell J, Pijl EM. An overview of forensic drug testing methods and their suitability for harm reduction point-of-care services. Harm reduction journal. 2017 Dec;14(1):1-3.

12. McDonald K, Maghsoudi N, Thompson H, Werb D. What's in Toronto's Drug Supply? [Internet]. Toronto, ON: Centre on Drug Policy Evaluation; 2020 [cited May 2021]. https://drugchecking.cdpe.org/

13. Tupper KW, McCrae K, Garber I, Lysyshyn M, Wood E. Initial results of a drug checking pilot program to detect fentanyl adulteration in a Canadian setting. Drug and alcohol dependence. 2018 Sep;190:242-5.

14. Guina J, Merrill B. Benzodiazepines I: upping the care on downers: the evidence of risks, benefits and alternatives. Journal of clinical medicine. 2018 Feb;7(2):17.

15. Kang M, Galuska MA, Ghassemzadeh S. Benzodiazepine Toxicity [Internet]. In: StatPearls. Treasure Island (FL): StatPearls Publishing; 2019 [cited May 2021]. https://www.ncbi.nlm.nih.gov/books/NBK482238/.

16. Gevorkyan J, Kinyua J, Pearring S, Rodda LN. A Case Series of Etizolam in Opioid-Related Deaths. Journal of Analytical Toxicology. 2021 Feb; https://doi.org/10.1093/jat/bkaa146

17. Toronto Public Health. Increase in overdoses observed at The Works on February 26, 2020 [Internet]. Toronto, ON: Toronto Public Health; 2020 [cited May 2021]. https://www.toronto.ca/wpcontent/uploads/2020/02/9477-Alert_Increase-in-overdoses-on-February-26_2020.pdf

18. Riederer AM, Campleman SL, Carlson RG, Boyer EW, Manini AF, Wax PM, Brent JA. Acute poisonings from synthetic cannabinoids-50 US Toxicology Investigators Consortium registry sites, 2010-2015. MMWR. Morbidity and mortality weekly report. 2016;65(27):692. 
19. Dasgupta N, Beletsky L, Ciccarone D. Opioid crisis: no easy fix to its social and economic determinants. American journal of public health. 2018 Feb;108(2):182-6.

20. Tyndall M. An emergency response to the opioid overdose crisis in Canada: a regulated opioid distribution program. CMAJ. 2018 Jan 15;190(2):E35-6.

21. Gomis B. How the Illicit Drug Trade is Adapting to Coronavirus Pandemic [Internet]. World Politics Review; 2020 [cited May 2021]. https://www.worldpoliticsreview.com/articles/28696/how-the-illicitdrug-trade-is-adapting-to-the-coronavirus-pandemic

\section{Figures}

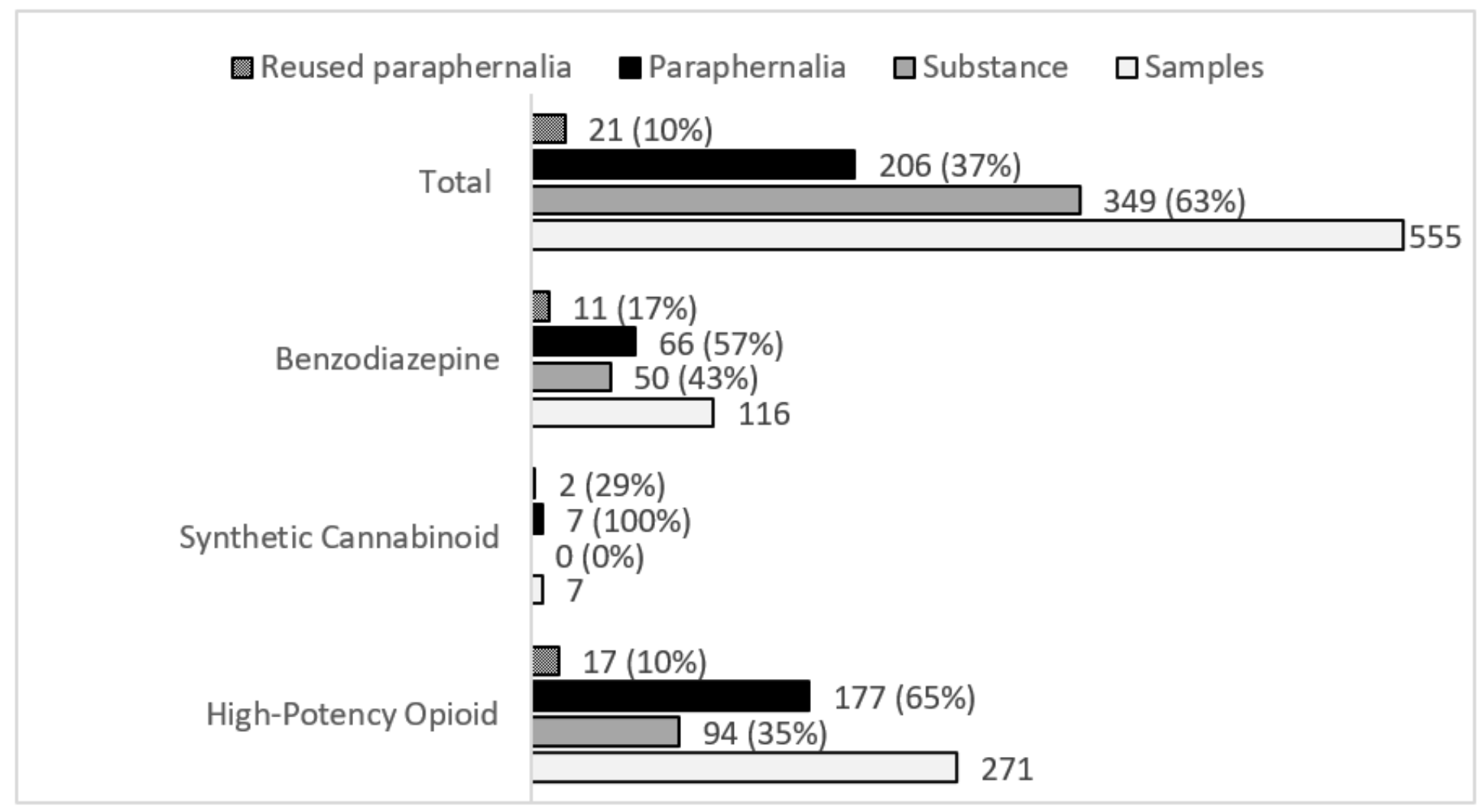

\section{Figure 1}

Graphical depiction of sample types stratified by category of drug detected (i.e., benzodiazepine, synthetic cannabinoid, or high-potency opioid) compared to total number of samples in that category $(\mathrm{N}=555)$ 


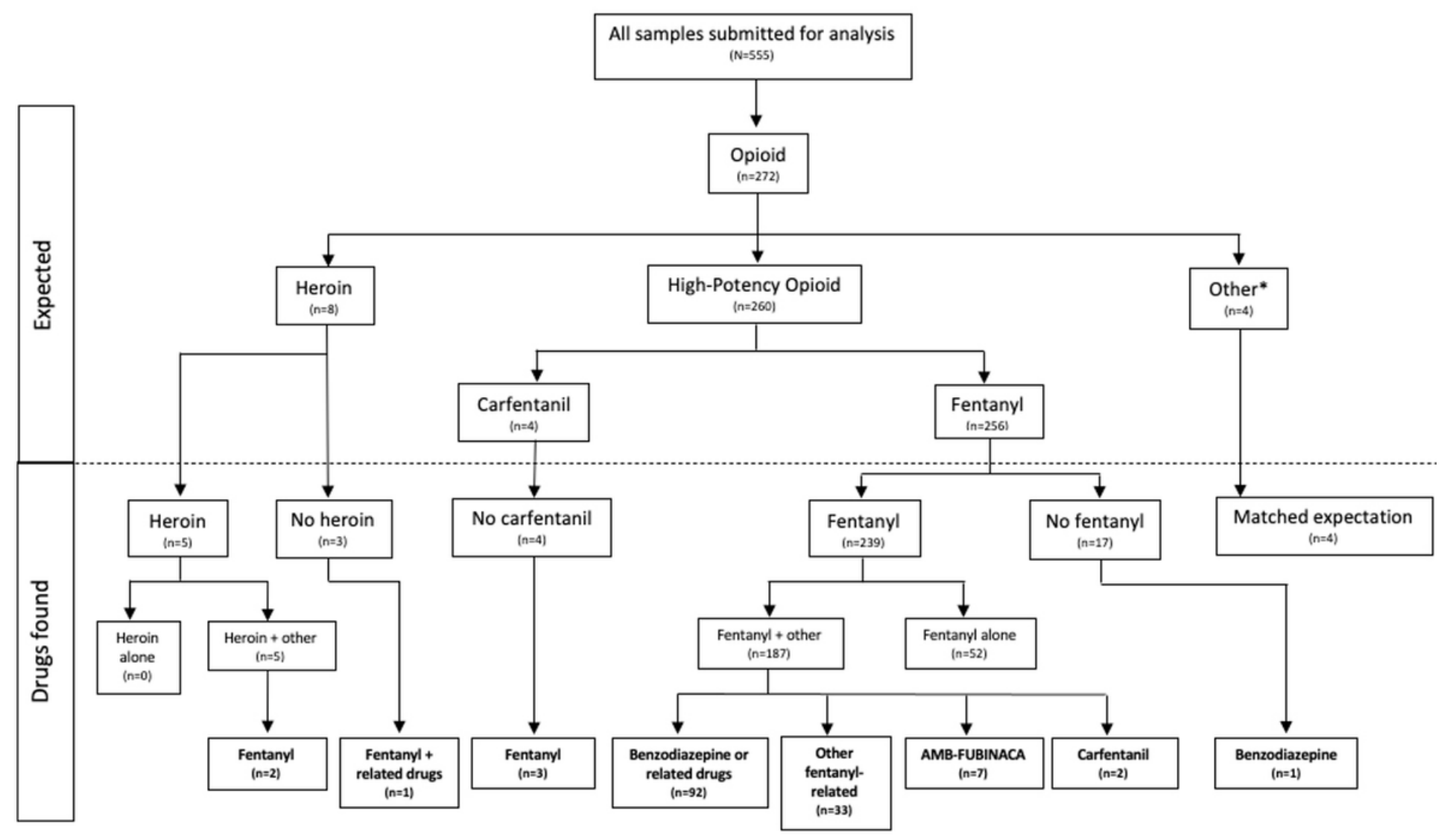

*Other: Oxycodone (3), O-Desmethyltramadol (1)

Figure 2

A breakdown of detected high-potency opioids, benzodiazepines or related drugs, and synthetic cannabinoids as drugs found in samples expected to contain variable opioids $(n=272)$ 